\title{
Problems in Coordinating and Accessing Primary Care for Attached and Unattached Patients Exacerbated During the COVID-19 Pandemic Year (the PUPPY Study): Protocol for a Longitudinal Mixed Methods Study
}

Emily Gard Marshall ${ }^{1}$, BA, MSc, PhD; Mylaine Breton ${ }^{2}, \mathrm{PhD}$; Benoit Cossette ${ }^{2}$, PhD; Jennifer Isenor ${ }^{3,4}$, PharmD; Maria Mathews ${ }^{5}$, PhD; Caitlyn Ayn ${ }^{1}$, MA; Mélanie Ann Smithman ${ }^{2}, \mathrm{MSc}$; David Stock ${ }^{1}$, PhD; Eliot Frymire ${ }^{6}$, MA; Lynn Edwards ${ }^{7}$, MHSA; Michael Green ${ }^{6,8,9}$, MD

\footnotetext{
${ }^{1}$ Primary Care Research Unit, Dalhousie Family Medicine, Halifax, NS, Canada

${ }^{2}$ Department of Community Health Sciences, Université de Sherbrooke, Longueuil, QC, Canada

${ }^{3}$ College of Pharmacy, Dalhousie University, Halifax, NS, Canada

${ }^{4}$ Department of Community Health and Epidemiology, Dalhousie University, Halifax, NS, Canada

${ }^{5}$ Department of Family Medicine, Schulich School of Medicine and Dentistry, Western University, London, ON, Canada

${ }^{6}$ Department of Family Medicine, Queen's University, Kingston, ON, Canada

${ }^{7}$ Nova Scotia Health, Halifax, NS, Canada

${ }^{8}$ Department of Public Health Sciences, Queen's University, Kingston, ON, Canada

${ }^{9}$ School of Policy Studies, Queen's University, Kingston, ON, Canada
}

\section{Corresponding Author:}

Emily Gard Marshall, BA, MSc, PhD

Primary Care Research Unit

Dalhousie Family Medicine

1465 Brenton Street Suite 402

Halifax, NS, B3J 3T4

Canada

Phone: 19024734155

Email: emily.marshall@dal.ca

\section{Abstract}

Background: The COVID-19 pandemic has significantly disrupted primary care in Canada, with many walk-in clinics and family practices initially closing or being perceived as inaccessible; pharmacies remaining open with restrictions on patient interactions; rapid uptake of virtual care; and reduced referrals for lab tests, diagnostics, and specialist care.

Objective: The PUPPY Study (Problems in Coordinating and Accessing Primary Care for Attached and Unattached Patients Exacerbated During the COVID-19 Pandemic Year) seeks to understand the impact of the COVID-19 pandemic across the quadruple aims of primary care, with particular focus on the effects on patients without attachment to a regular provider and those with chronic health conditions.

Methods: The PUPPY study builds on an existing research program exploring patients' access and attachment to a primary care practice, pivoted to adapt to the emerging COVID-19 context. We intend to undertake a longitudinal mixed methods study to understand critical gaps in primary care access and coordination, as well as compare prepandemic and postpandemic data across 3 Canadian provinces (Quebec, Ontario, and Nova Scotia). Multiple data sources will be used such as a policy review; qualitative interviews with primary care policymakers, providers (ie, family physicians, nurse practitioners, and pharmacists), and patients $(\mathrm{N}=120)$; and medication prescriptions and health care billing data.

Results: This study has received funding by the Canadian Institutes of Health Research COVID-19 Rapid Funding Opportunity Grant. Ethical approval to conduct this study was granted in Ontario (Queens Health Sciences \& Affiliated Teaching Hospitals Research Ethics Board, file 6028052; Western University Health Sciences Research Ethics Board, project 116591; University of Toronto Health Sciences Research Ethics Board, protocol 40335) in November 2020, Québec (Centre intégré universitaire de 
santé et de services sociaux de l'Estrie, project 2020-3446) in December 2020, and Nova Scotia (Nova Scotia Health Research Ethics Board, file 1024979) in August 2020.

Conclusions: To our knowledge, this is the first study of its kind to explore the effects of the COVID-19 pandemic on primary care systems, with particular focus on the issues of patient's attachment and access to primary care. Through a multistakeholder, cross-jurisdictional approach, the findings of the PUPPY study will inform the strengthening of primary care during and beyond the COVID-19 pandemic, as well as have implications for future policy and practice.

International Registered Report Identifier (IRRID): DERR1-10.2196/29984

(JMIR Res Protoc 2021;10(10):e29984) doi: 10.2196/29984

\section{KEYWORDS}

primary care; health services research; health policy; mixed methods research; COVID-19; protocol; policy; longitudinal; coordination; access; impact; virtual care; virtual health; Canada

\section{Introduction}

\section{Pre-COVID-19 Challenges in Canadian Primary Care}

More than $75 \%$ of visits in Canada are within a primary care setting [1]. Access to primary care is the foundation of a strong health care system; it is vital to achieving the quadruple aim of (1) enhancing patient experience, (2) promoting care team well-being, (3) improving population health, and (4) optimizing costs by managing health in primary care through the patient's life course and thereby reducing the burden on acute care [2]. Primary care includes comprehensive and routine care, health promotion, disease prevention, diagnosis and treatment of illness and injury, coordination of care with other specialists, among other care services.

Even prior to the COVID-19 pandemic, Canadians reported lower access to a source of regular primary care than individuals residing in other Commonwealth nations, with only $90 \%$ indicating a regular physician and/or place to receive care in 2020 [3]. Access to a regular source of care in Canada, traditionally with family physicians or nurse practitioners, has declined in recent years, and it varies widely across provinces $[3,4]$. Individuals without a regular primary care provider are classified as "unattached patients" [5,6], and they typically experience poorer health outcomes and less appropriate care than patients with access to a regular primary care provider (ie, attached patients) [7,8]. Vulnerable patients and those with complex needs, including those with low-income levels and/or low social support, are less likely to be attached to a primary care provider, despite the fact that they would benefit more from access to comprehensive and continuous primary care than less vulnerable patients $[7,9,10]$. Unattached patients are less likely to seek the needed care and more frequently use alternative points of access, such as walk-in clinics, than attached patients [11].

As Canadian provinces struggle to support patient attachment to primary care, specific types of care may be provided by community-based pharmacists in some jurisdictions $[12,13]$. However, primary care provided by pharmacists may not be sufficient and recommended for all patients, particularly those with chronic or complex health concerns and those with needs outside of pharmacists' legislated scope. Because of these challenges in accessing and being attached to a regular primary care provider, many Canadians rely on emergency departments or walk-in clinics to receive care. Among Canadians surveyed in $2020,42 \%$ reported that they had visited an emergency department within the previous 2 years, and among those respondents, $40 \%$ indicated their concern could have been treated by a regular primary care provider [3]. Because having access to a regular primary care provider has been shown to reduce the likelihood of emergency department use [14], promoting patient attachment to primary care was a key priority prior to the onset of the COVID-19 pandemic. Most Canadian provinces have therefore developed strategies including centralized waitlists for unattached patients and dedicated clinics to address this concern $[15,16]$.

\section{Early Findings Regarding the Effects of the COVID-19 Pandemic on Primary Care}

The COVID-19 pandemic has caused unprecedented disruption to primary care in Canada and internationally. During the peak of the first wave of COVID-19 in Canada, many primary care clinics reduced their work hours [17], leaving patients and caregivers fearful and uncertain about how to access care. Primary care providers were required to make rapid shifts in practice to comply with infection prevention and control requirements, incorporate COVID-19 triage and nonacute case management, address reduced referral and diagnostics access, and implement virtual care where possible [18-21]. Primary care providers also had to engage in practice redesign, secure access to personal protective equipment, and integrate changes in scope of practice in the case of pharmacists in some jurisdictions [22]. Many primary care providers were also redeployed or prepared to be redeployed to COVID-19 testing and treatment roles [21,23].

Health care access is defined as "the opportunity to have health care needs fulfilled" [24]. Access to health care is influenced by (1) the accessibility of providers, organizations, institutions and systems; and (2) the ability of individuals, households, communities, and populations to access primary care. These influential elements have had a COVID-19 "anvil" dropped on their capacity to provide, and access, primary care (see Figure 1).

The COVID-19 pandemic led to delayed and forgone care, concurrent with increased mental health needs of providers and patients. As the pandemic continues, there are anticipated waves of COVID-19 fallout (Figure 2) [25,26]. Although emerging evidence illustrates some significant impacts of the pandemic 
on primary care systems globally [17], the effects of the pandemic on patient attachment and access to primary care remain unclear. There is also mounting evidence on the impact of the COVID-19 pandemic on patient and provider well-being [3].

Figure 1. Depiction of the COVID-19-induced disruption to the accessibility of the health care system and the ability of patients to access the system (adapted from Levesque et al [24]).

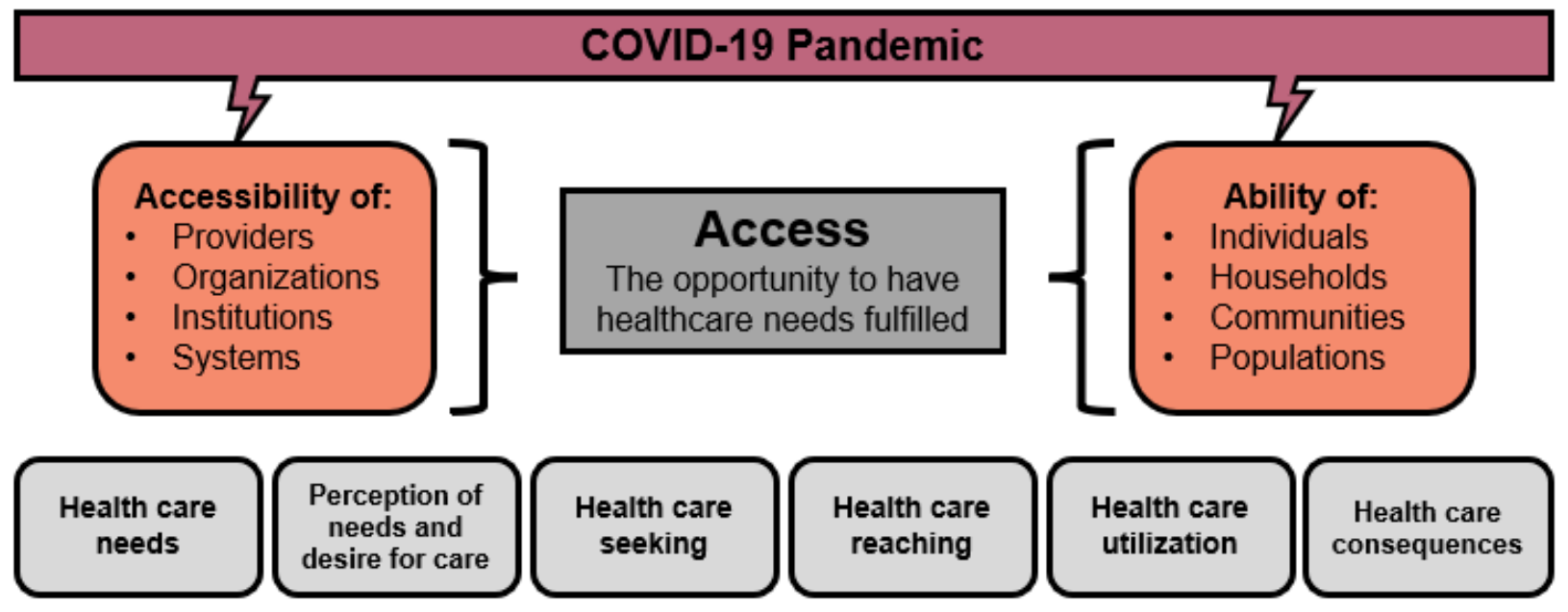

Figure 2. Anticipated waves of the COVID-19 pandemic on primary care.

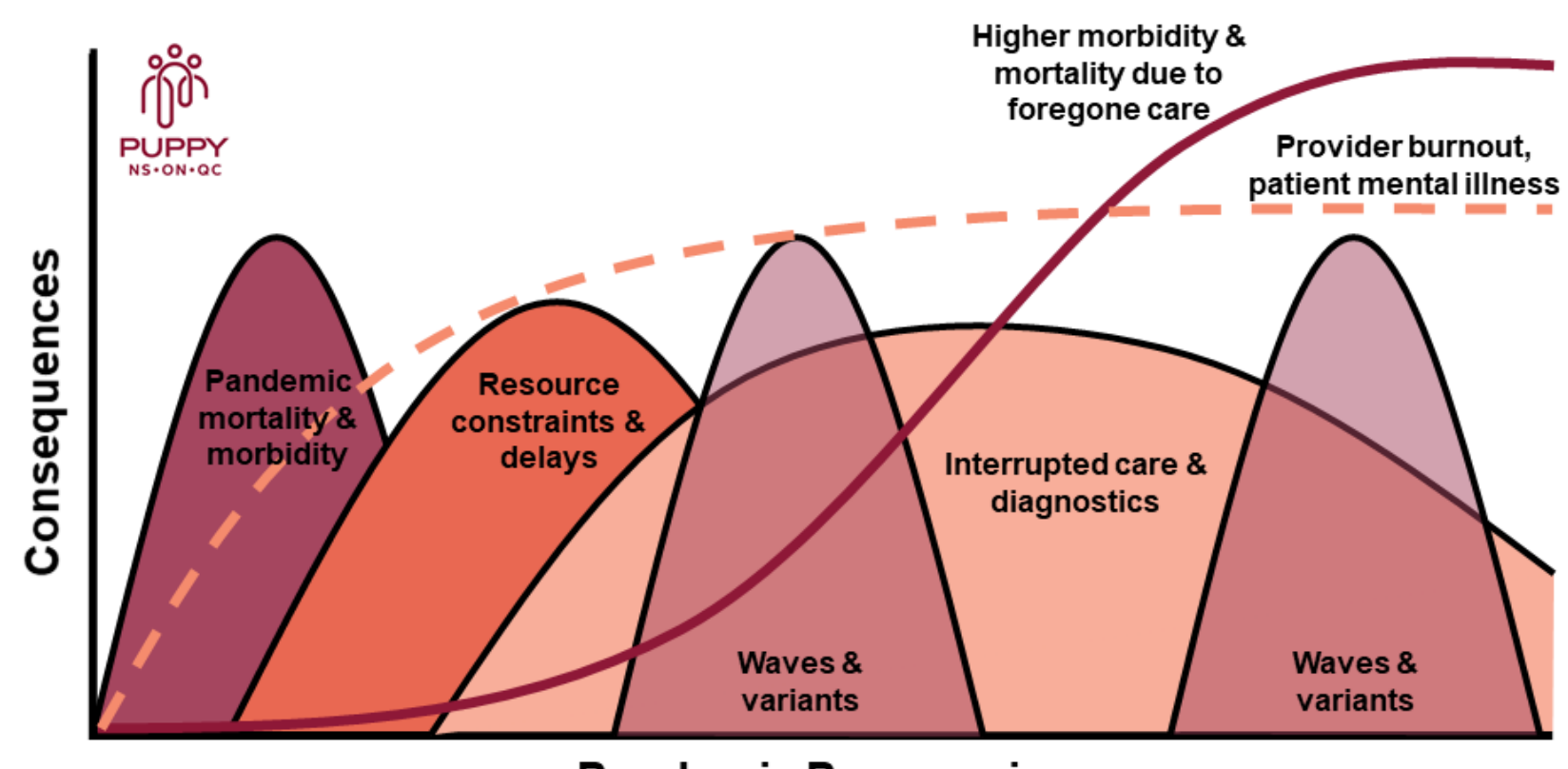

Pandemic Progression

\section{Pivoting a Program of Primary Care Research to Address the COVID-19 Crisis}

At the onset of the COVID-19 pandemic, many health research studies in Canada were required to halt immediately while a pandemic plan and appropriate public health measures were created and enacted. For example, our cross-provincial CUP study (Comparative Analysis of Centralized Waitlist Effectiveness, Policies, and Innovations for Connecting Unattached Patients to Primary Care Providers), funded by the Canadian Institutes of Health Research (CIHR), examining pre-COVID-19 patient attachment to primary care in 3 provinces, namely Ontario (ON), Québec (QC), and Nova Scotia (NS), was put on hold for several months [27].

As the pandemic continued, our team recognized that the existing research aims and methods would not be sufficient to address the effects in the novel COVID-19 context. Furthermore, new research questions were emerging rapidly due to changes in the policy landscape and provider roles in primary care systems across Canada. For these reasons, it was necessary to pivot existing studies to include pandemic-specific analyses and capture changes in primary care systems over time, while identifying novel ways of data collection in a safe manner during the pandemic. Our research team rapidly engaged with our study 
team, which included the departments and ministries of health, health authorities, primary care providers and their organizations, and our patient partners. Through these consultations conducted in March and April 2020, we quickly gathered lists of key concerns and priority areas and synthesized and thematically grouped them. The co-principal investigators (EGM, MB, MG, JEI, MM, and BC) then developed new strategies for answering emerging questions and updated the study methods to reflect the new COVID-19 primary health care landscape and ability to work safely. This newly expanded and updated protocol was then submitted for funding in May 2020 .

\section{Objectives}

This study will identify and evaluate strategies to provide primary care access and COVID-19 triage and care by family physicians, nurse practitioners, and pharmacists that can be scaled-up to promote attachment and improved access for patients across and beyond the COVID-19 waves. We will focus particularly on patients who are unattached, with complex care needs, and/or experiencing social barriers to care, as primary care-based support for these populations may lead to better outcomes for these patients and the health care system across the quadruple aim. Accordingly, the study objectives are as follows:

1. To identify primary care policies and interventions implemented in response to the COVID-19 pandemic and to describe how they affect primary care attachment (ie, demand) and accessibility (ie, supply).

2. To understand how COVID-19-related changes affect: (1) patients' experience of accessing primary care, considering different needs, identity factors (eg, age, gender) and access abilities (unattached and attached patients and/or patients with complex needs), and (2) provider health and well-being.

3. To determine how these pandemic-related changes have impacted health care utilization, attachment to primary care providers, and medication prescription, as indicators of access to primary care: We hypothesize that unattached patients and those with chronic conditions are vulnerable to poorer primary care access and health outcomes exacerbated during the COVID-19 pandemic.

4. To share promising strategies to provide access to primary care involving policymakers, primary care providers, and patients across Canada in the immediate, intermediate, and reflective phases of the pandemic.

\section{Methods}

\section{Study Design and Setting}

To address the rapid effects of the COVID-19 pandemic on policy, practice, and patient access to primary care in line with the objectives described above, a longitudinal mixed-methods observational study building from our team's ongoing research is being conducted. Data will be collected and compared across 3 Canadian provinces (NS, QC, and $\mathrm{ON}$ ) by using four different methods (see Figure 3) with integration. Data collection will include (1) a content analysis of policies affecting primary care access in the wake of the COVID-19 pandemic; (2) qualitative interviews with providers, patients, and policymakers; (3) surveys of providers and patients; and (4) analysis of administrative data, including centralized waitlists, billing, and prescribing data, to track health care access and utilization, and primary care provider prescribing patterns before, during, and after the COVID-19 pandemic.

Figure 3. Overview of study objectives, methods, and relationships between study activities. QUAL: qualitative analysis; QUAN: quantitative analysis.

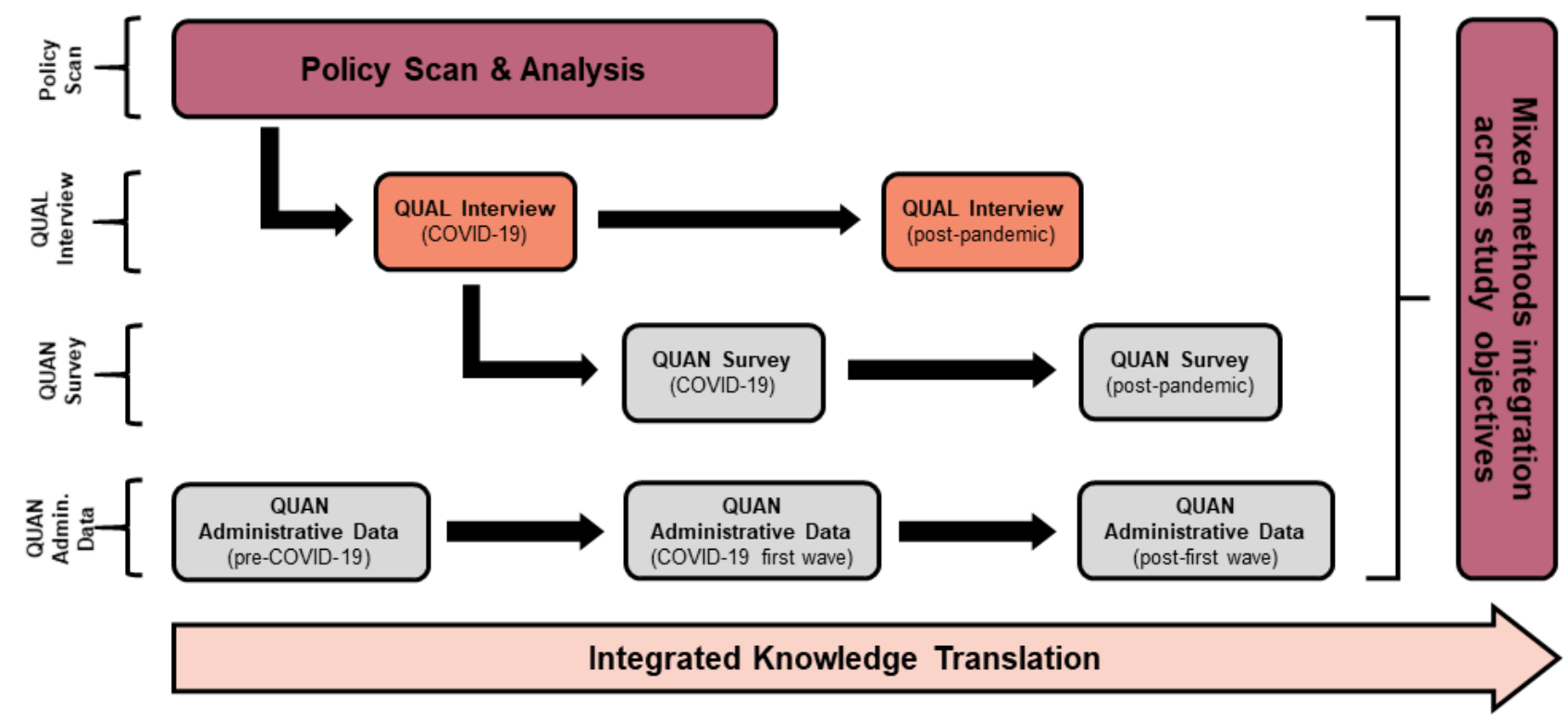

\section{Study Participants}

A purposeful sampling approach will be applied to include participant representation from key stakeholders in primary care access, including policymakers, patients, and primary care providers via qualitative interviews, surveys, and linked administrative health data (see Table 1). Although providers from many professions contribute to primary care across 
systems, our study will focus on family physicians, nurse practitioners, and community pharmacists. The inclusion of the latter is due to the growing number of publicly-funded services offered by pharmacists in several Canadian jurisdictions in recent years, with limited evaluation (eg, prescription for minor ailments, immunizations, reviewing and managing medications) [28-31], which establishes more primary care access options.

Table 1. Participant groups involved in each of the four data collection methods.

\begin{tabular}{lllll}
\hline Participant group & \multicolumn{2}{l}{ Data collection method } & & Knowledge Translation \\
& Qualitative interviews & Quantitative surveys & Policy content analysis & Administrative data \\
\hline Patients & $\checkmark$ & $\checkmark$ & $\checkmark$ & $\checkmark$ \\
Providers & $\checkmark$ & $\checkmark$ & $\checkmark$ & $\checkmark$ \\
Policymakers & $\checkmark$ & $\checkmark$ & $\checkmark$ & $\checkmark$ \\
\hline
\end{tabular}

\section{Policy Content Analysis}

Contextual factors affecting primary care access will be identified through extensive provincial policy reviews and interviews with health authority, government, regulator, and corporate policymakers. The unit of analysis considered is provincial. We will document primary care changes in a context that coincides with key developments related to the COVID-19 pandemic to inform recommendations for transformation, scale, and spread. Provincial policies may include provider hiring and funding; delivery models, including the rapid deployment of virtual care modalities across Canada; incentives, programs, and innovations to aid patient access; fulfilling the needs of unattached and other vulnerable patients; and other policies that may play moderating roles in primary care (eg, provider well-being). We will focus on influential policies, where policies are defined by the World Health Organization as "decisions, plans, and actions... undertaken to achieve specific healthcare goals" and identified contextual factors [32].

The Tomoaia-Cotisel approach [33] for assessing and reporting contextual factors of primary care innovations will be applied to the qualitative and policy content analyses components of the study. The framework involves engaging diverse perspectives, considering multiple policy and context levels, time, formal and informal system/culture, and identifying interactions between policies and contexts. It is tailored specifically to innovations in primary care and considers moderators at multiple levels.

\section{Qualitative Interviews}

Qualitative methods are designed to elicit experiences and perceptions of phenomena where little is known-an ideal approach to study the impact of the COVID-19 pandemic on primary care. Stakeholder groups to be interviewed include patients, family physicians who do and do not accept new patients, nurse practitioners, community pharmacists, and policymakers with roles relevant to primary care access and attachment. The proposed longitudinal data collection will support interviews, with 10 participants per stakeholder group per province (ie, $\mathrm{N}=120$ participants), which will ensure saturation [34,35].

Interviews will be conducted during and after the COVID-19 pandemic to elicit current and retrospective lived experiences. Interview guides will be developed to reflect key issues pertinent to stakeholders. For example, providers will be asked questions pertaining to changes in their practice. Patients will be asked about their experience with primary care changes and the impact of these changes on access to care and well-being. Policymakers will be invited to share processes for, and outcomes of, policy change and will be consulted on relevant documents to include in our policy content analysis (study objective 1).

Purposive and snowball sampling strategies will be used, with stratification by relevant participant characteristics (gender, rurality, practice characteristics, etc). Invitations for interview participants will be distributed via the provincial centralized waitlists, partnered organizations, and social media. We will iteratively revise our sampling and recruitment strategies as we collect data and learn more about patient and provider experiences [36].

Informed consent discussions and semistructured, in-depth interviews will be conducted virtually using Zoom videoconferencing software (Zoom Video Communications Inc.) by a Masters-trained researcher. Audio recordings of interviews will be transcribed verbatim and coded in NVivo software (QSR International). Coding reports will be generated and examined to uncover themes and patterns in the data.

Preliminary thematic analysis will provide rapid reporting to stakeholders. A framework analysis [37] approach will incorporate the conceptual framework proposed by Levesque et al [24] for access to health care and be implemented across study phases for comparative analysis. This method allows for inductive and deductive coding approaches [37]. We will code deductively using Levesque's framework and inductively using interview transcripts, thereby allowing emergent themes to enhance what can be gleaned from the framework alone. Intraand cross-case analysis will be conducted by incorporating provincial framework analysis matrices [37,38].

\section{Quantitative Surveys}

Brief surveys for patients and providers will be developed to determine the prevalence of our emerging qualitative themes. Surveys designed for providers will be delivered via the secure web-based Opinio survey tool (Objectplanet, Inc.) in the postpandemic period, to measure the degree to which COVID-19-related policy changes have affected primary care access and attachment, as well as their personal wellness. Recruitment support will be provided by our partners. A web-based patient survey at the same time point will explore patient primary care access and attachment during the 
COVID-19 pandemic. A convenience sample of 1000 patient respondents per province $(\mathrm{N}=3000)$ will be recruited using a third-party survey sampling company. It is estimated that a sample size of 1000 per province would permit adequate segment sizes for comparison of results among patient groups and provinces. The use of third-party sampling services is common for health care research involving the general public [39]. Bivariable and multiple regression models will be generated to show trends and associations on key elements across phases. Follow-up surveys will be conducted at a later point to assess changes over time.

\section{Administrative Data}

Analyses of pre-COVID-19 prescription dispensation, centralized waitlist, physician billing, and inpatient and outpatient hospital discharge data has already begun to examine the effectiveness of centralized waitlists for a related study [27]. As part of the PUPPY study, we will expand this analysis to explore changes across the pre-COVID-19, COVID-19, and postpandemic periods. Harmonized indicators of health care utilization (eg, primary care, emergency, hospitalization, and potentially avoidable inpatient care), primary care attachment indicators (primary care provider attachment and continuity of primary care), and primary care service provision (eg, frequency and type of primary care encounters and continuity of medication dispensation for maintenance of chronic conditions) will be measured across the 3 participating provinces. Change in these indicators, and in care continuity, will be estimated and compared across pandemic wave-indexed study periods.

Multivariable regression will be used to identify potential clinical (eg, patient complexity and comorbidity), demographic, and socioeconomic determinants of primary care need, as well as changes in these indicators over the course of the pandemic. Socioeconomic determinants are derived from the 2016 Canadian census data, including the Canadian Index of Multiple Deprivation, with a focus on dimensions of economic dependency, ethnocultural composition, and situational vulnerability [27]. Centralized waitlist data will be used to measure primary care attachment and assess changes in access to primary care. Building on ongoing work, variation in patient primary care provider attachment rates, demand for attachment, and time to attachment among those patients identified on centralized wait lists will be quantified. Moreover, the changes in these outcomes will be assessed across study periods.
Determinants of these outcomes will be identified, and their relative magnitude will be estimated using multivariable techniques. In each province, study populations will be stratified by age, sex (and gender where feasible), degree of comorbidity and geography (ie, urban vs rural) to identify those at greatest risk of being unattached to a primary care provider.

\section{Mixed Methods Integration}

As a longitudinal evaluation comparing 3 provincial cases, the study will use a series of mixed methods integration approaches and principles to inform the planning, analysis, and interpretation across the four data types [40]. Adapted from Goldsmith et al [41], Figure 4 provides a depiction of the ways in which the four study methods will inform one another and ultimately lead to meta-inferences strengthened by this mixed methods approach.

In the planning phase, qualitative work and policy content analysis approaches will be conducted in parallel, with findings from each iteratively informing data collection and planning for the other. For example, qualitative interviews will support identification of policy documentation unable to be identified through traditional searches, whereas analysis of policy documents will uncover areas of interest to explore future qualitative interviews. Additionally, in an exploratory sequential approach, qualitative interview findings will be used to inform development of a quantitative survey to build upon and explore the breadth and depth of perceptions expressed by interview participants.

In the analysis phase, data will be collected through embedding and merging - a process by which multiple datasets are collated for analysis and triangulation via iterative comparison [40]. This process will enable creation of rich case descriptions. In particular, a timeline for each case (province) illustrating the patterns in data alongside policy milestones and insight into relevant participant experiences will be developed. The frameworks being used to inform our approaches have been used across multiple methodologies and mixed methods study designs and will facilitate these comparisons [24,33]. As shown in Figure 4, the use of congruent methods will allow numerous comparisons between datasets for both primary and emergent research questions. Mixed methods interpretation will be conducted via the creation of mixed methods narratives and joint displays from which meta-inferences incorporating multiple methodologies can be generated [40]. 
Figure 4. Summary of mixed methods integration approaches across the planning, analysis, and interpretation stages of the PUPPY study. QUAL: qualitative analysis; QUAN: quantitative analysis.

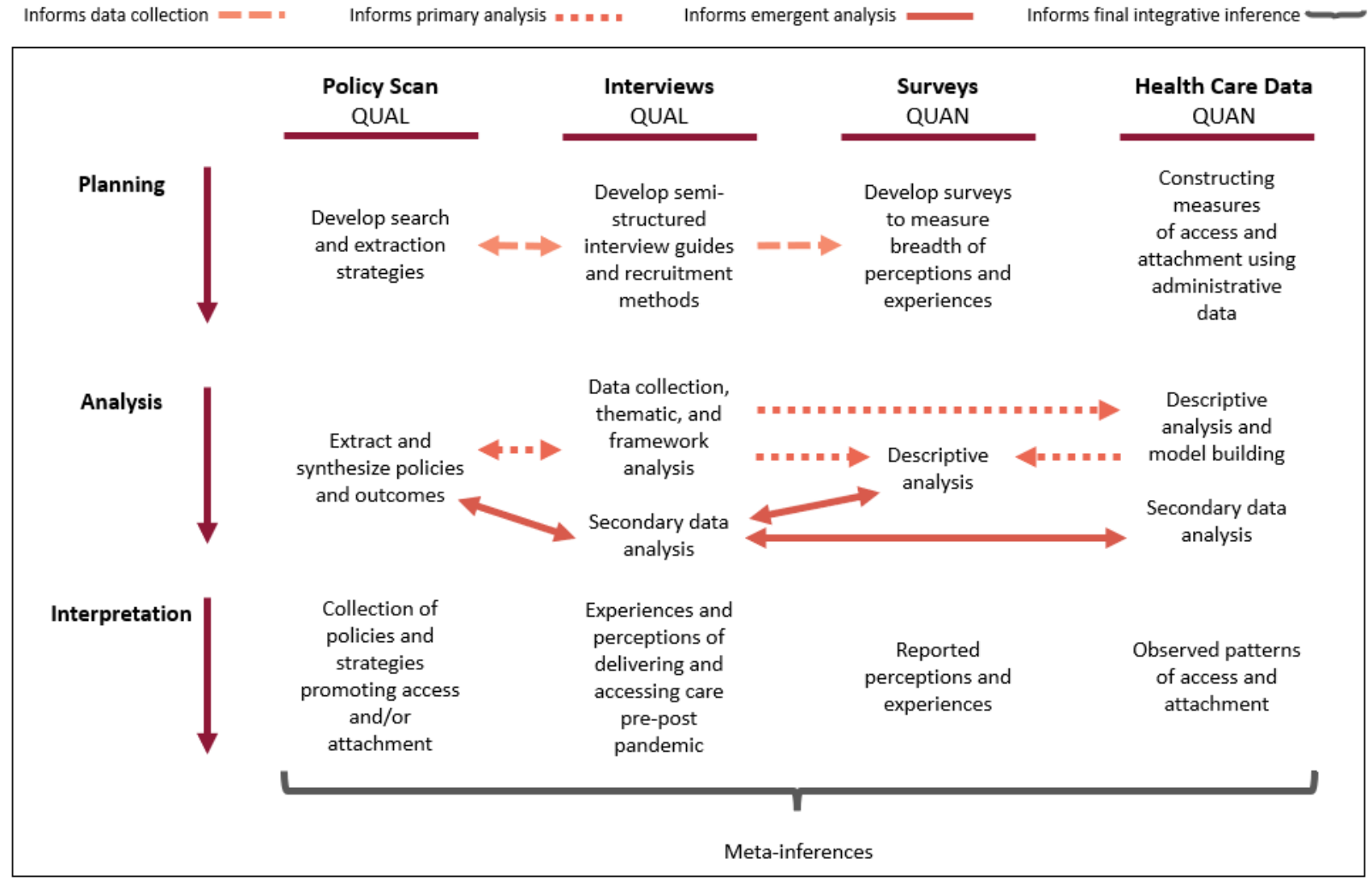

\section{Results}

\section{Funding}

In June 2020, our study team received funding through the CIHR COVID-19 Rapid Funding. The funding opportunity encouraged an expansion of ongoing studies to expedite the translation of findings and offered resources to identify and incorporate emerging research questions, expand existing methods, and include additional methods where necessary. Through this opportunity, our team received the resources necessary to undertake this study to elucidate the effects of the COVID-19 pandemic on primary care in Canada. Ethics approval was received in Quebec in December 2020, in Ontario in November 2020, and in Nova Scotia in August 2020.

\section{Ethics}

Approval to conduct this study was granted in the Canadian provinces of Ontario (Queens Health Sciences \& Affiliated Teaching Hospitals Research Ethics Board, file number 6028052; Western University Health Sciences Research Ethics Board, project 116591; University of Toronto Health Sciences Research Ethics Board, protocol number 40335), Québec (Centre intégré universitaire de santé et de services sociaux de l'Estrie, project number 2020-3446), and Nova Scotia (Nova Scotia Health Research Ethics Board, file number 1024979). As the PUPPY study builds upon and expands the timeline of ongoing research projects, including the CUP study [27], ethical approvals have in several cases been granted as amendments or extensions to the CUP study to facilitate rapid implementation of study activities.

\section{Timeline}

Data collection for the PUPPY study will take place in 2021-2022, with rapid reporting between 2021 and 2023. As of April 2021, recruitment for qualitative interviews has begun in NS and QC, with recruitment expected to take place in $\mathrm{ON}$ when COVID-19-related constraints have eased. Each province is in the process of accessing administrative health data and linking it to provincial centralized waitlist data. Integrated and end-of-grant knowledge translation of the PUPPY study and subsequent research will follow up on key areas identified.

\section{Discussion}

\section{Partnership and Knowledge Translation}

Our team includes regulatory bodies and associations representing family physicians, nurse practitioners, and community pharmacists, as well as support from the CIHR's Strategy for Patient Oriented Research (SPOR) Primary and Integrated Healthcare Innovations (PIHCI) Networks and the SPOR Support for Patient-Oriented Research and Trials Units to aid data collection and knowledge dissemination. Guidance from COVID-19 policymaking partners will ensure relevance and uptake while minimizing the burden of study activities on participants, which is particularly critical given the high demands of the pandemic on all stakeholders involved in our study. Data collection activities will occur remotely to comply with public health measures. In anticipation of possible participant distress and recognizing the impact of the pandemic on mental health and wellness generally, we will provide a list 
of resources to appropriately trained mental health and primary care providers.

To ensure appropriate dissemination and translation of study findings, all data collection begins with consultation. Team members representing all stakeholder groups, including providers, policymakers, and patients, will participate in the development and refinement of study tools, analysis, interpretation, and dissemination plans. Knowledge dissemination will include multiple modalities to maximize the uptake of findings. Policy briefs and reports will be shared at each study phase and will be assisted by professional graphic and communication design support. Other modalities include peer-reviewed publications, conference presentations, local presentations to key stakeholder groups (eg, provider associations, health authorities, departments or ministries of health, and primary care provincial leadership meetings), knowledge sharing on departmental websites, blog posts, and social media. Team members, including patient partners, will have the opportunity to inform, author, and participate in dissemination activities. Using CUP study funds in the post-pandemic period, we will facilitate cross-jurisdictional learning via a symposium with stakeholders from across Canada to improve primary care attachment and to manage patients within and outside of pandemics.

\section{Conclusions}

The PUPPY study is designed to provide rapid support for primary care policymaking, provider needs, and patient access to primary care based on the investigation across the various COVID-19 waves. We will regularly communicate emerging recommendations to our partners for timely policy optimization. Immediate-term early data collection will provide feedback on new policies in primary care settings and effects on patient access, thereby providing insight into possible unintended consequences of rapid policy transformation and revealing promising strategies. This information will inform provision of care through changing pandemic contexts, including requirements for physical distancing and safety requirements. In the intermediate term, our study will document changes in the primary care policy landscape to strengthen the response to additional "waves" related to COVID-19 outbreaks. Findings will be distributed to study partners and beyond via our networks (eg, CanCOVID, pan-Canadian PIHCI Networks, and North American Primary Care Research Group), to support cross-jurisdictional pandemic response. In the long term, the study findings will help us grasp the impact of these policy changes and events on the ability of systems and providers to coordinate and deliver primary care, patient access to primary care, and on health outcomes. Recommended best practices to improve access to primary care as we transition to a post-pandemic context will be widely shared with our partners via our knowledge dissemination plan as outlined above.

\section{Acknowledgments}

We would like to acknowledge the generous support of the organizational partners and team members, including the academic researchers, providers, policymakers, and patient partners (Sarah Peddle, Ana Correa Woodrow, Nicole Desjardins, and Danièle Roberge). A full list of our team members and partners can be found on the study website [42]. We would like to thank the Canadian Institutes of Health Research for their support in the form of a COVID-19 Rapid Funding Opportunity Grant (grant 447605).

\section{Authors' Contributions}

The study was conceived by the lead author (EGM) who also led the writing of the manuscript with active assistance from the coauthors. All authors made substantive contributions to the conception and design of the study, which includes qualitative, quantitative, mixed methods, patient engagement, and knowledge translation components. All authors have critically reviewed this manuscript and approve the version to be published.

\section{Conflicts of Interest}

None declared.

\section{References}

1. Primary Health Care (PHC) Indicators Chartbook: An Illustrative Example of Using PHC data for Indicator Reporting Internet. Canadian Institute for Health Information. URL: https://www.cihi.ca/en/ primary-health-care-phc-indicators-chartbook-an-illustrative-example-of-using-phc-data-for-indicator [accessed 2021-02-16]

2. Bodenheimer T, Sinsky C. From triple to quadruple aim: care of the patient requires care of the provider. Ann Fam Med 2014 Nov 10;12(6):573-576 [FREE Full text] [doi: 10.1370/afm.1713] [Medline: 25384822]

3. How Canada Compares: Results From the Commonwealth Fund's 2020 International Health Policy Survey of the General Population in 11 Countries. Canadian Institute of Health Information. 2021. URL: https://www.cihi.ca/sites/default/files/ document/how-canada-compares-cmwf-survey-2020-meth-note-en.pdf [accessed 2021-09-28]

4. Schoen C, Osborn R, Doty MM, Bishop M, Peugh J, Murukutla N. Toward higher-performance health systems: adults' health care experiences in seven countries, 2007. Health Aff (Millwood) 2007;26(6):w717-w734. [doi:

10.1377/hlthaff.26.6.w717] [Medline: 17978360] 
5. Access to a regular medical doctor, 2013. Statistics Canada. 2015. URL: https://www150.statcan.gc.ca/n1/pub/82-625-x/ 2014001/article/14013-eng.htm [accessed 2020-07-30]

6. Breton M, Wong S, Smithman M, Kreindler S, Jbilou J, Marshall E, et al. Centralized waiting lists for unattached patients in primary care: learning from an intervention implemented in seven Canadian provinces. Healthc Policy 2018 May;13(4):65-82 [FREE Full text] [doi: 10.12927/hcpol.2018.25555] [Medline: $\underline{\text { 30052190] }}$

7. Burge F, Haggerty J, Pineault R, Beaulieu M, Lévesque JF, Beaulieu C, et al. Relational continuity from the patient perspective: comparison of primary healthcare evaluation instruments. Healthc Policy 2011 Dec;7(Spec Issue):124-138 [FREE Full text] [Medline: 23205040]

8. McIsaac W, Fuller-Thomson E, Talbot Y. Does having regular care by a family physician improve preventive care? Can Fam Physician 2001 Jan;47:70-76 [FREE Full text] [Medline: 11212436]

9. Olsen CG, Boltri JM, Amerine J, Clasen ME. Lacking a primary care physician is associated with increased suffering in patients with severe mental illness. J Prim Prev 2017 Dec;38(6):583-596. [doi: 10.1007/s10935-017-0490-7] [Medline: 28929367]

10. Crooks VA, Agarwal G, Harrison A. Chronically ill Canadians' experiences of being unattached to a family doctor: a qualitative study of marginalized patients in British Columbia. BMC Fam Pract 2012 Jul 16;13(1):69 [FREE Full text] [doi: 10.1186/1471-2296-13-69] [Medline: 22799280]

11. Randall E, Crooks VA, Goldsmith LJ. In search of attachment: a qualitative study of chronically ill women transitioning between family physicians in rural Ontario, Canada. BMC Fam Pract 2012 Dec 23;13(1):125 [FREE Full text] [doi: 10.1186/1471-2296-13-125] [Medline: 23259681]

12. Blondal AB, Jonsson JS, Sporrong SK, Almarsdottir AB. General practitioners' perceptions of the current status and pharmacists' contribution to primary care in Iceland. Int J Clin Pharm 2017 Aug;39(4):945-952. [doi: 10.1007/s11096-017-0478-7] [Medline: 28616683]

13. Manolakis P, Skelton J. Pharmacists' contributions to primary care in the United States collaborating to address unmet patient care needs: the emerging role for pharmacists to address the shortage of primary care providers. Am J Pharm Educ 2010 Dec 15;74(10):S7 [FREE Full text] [doi: 10.5688/aj7410s7] [Medline: 21436916]

14. Mian O, Pong R. Does better access to FPs decrease the likelihood of emergency department use? Results from the Primary Care Access Survey. Can Fam Physician 2012 Nov;58(11):e658-e666 [FREE Full text] [Medline: 23152473]

15. Breton M, Green M, Kreindler S, Sutherland J, Jbilou J, Wong ST, et al. A comparative analysis of centralized waiting lists for patients without a primary care provider implemented in six Canadian provinces: study protocol. BMC Health Serv Res 2017 Jan 21;17(1):60 [FREE Full text] [doi: 10.1186/s12913-017-2007-8] [Medline: 28109279]

16. Breton M, Smithman M, Vandesrasier A, Kreindler S, Sasseville M, Sutherland J, et al. Attaching patients in primary care through centralized waiting lists: seven Canadian provinces compared. HRO-ORS 2019 Mar 11;7(1) [REE Full text] [doi: 10.13162/hro-ors.v7i1.3773]

17. Huston P, Campbell J, Russell G, Goodyear-Smith F, Phillips R, van Weel C, et al. COVID-19 and primary care in six countries. BJGP Open 2020 Oct;4(4) [FREE Full text] [doi: 10.3399/bjgpopen20X101128] [Medline: $\underline{32900708]}$

18. Krist AH, DeVoe JE, Cheng A, Ehrlich T, Jones SM. Redesigning primary care to address the COVID-19 pandemic in the midst of the pandemic. Ann Fam Med 2020 Jul 13;18(4):349-354 [FREE Full text] [doi: 10.1370/afm.2557] [Medline: 32661037]

19. Duckett S. What should primary care look like after the COVID-19 pandemic? Aust J Prim Health 2020;26(3):207. [doi: 10.1071/py20095]

20. Rawaf S, Allen LN, Stigler FL, Kringos D, Quezada Yamamoto H, van Weel C, Global Forum on Universal Health CoveragePrimary Health Care. Lessons on the COVID-19 pandemic, for and by primary care professionals worldwide. Eur J Gen Pract 2020 Dec 28;26(1):129-133 [FREE Full text] [doi: 10.1080/13814788.2020.1820479] [Medline: 32985278 ]

21. Kearon J, Risdon C. The role of primary care in a pandemic: reflections during the COVID-19 pandemic in Canada. J Prim Care Community Health 2020 Sep 27;11:2150132720962871 [FREE Full text] [doi: 10.1177/2150132720962871] [Medline: 32985333]

22. Bragazzi N, Mansour M, Bonsignore A, Ciliberti R. The role of hospital and community pharmacists in the management of COVID-19: towards an expanded definition of the roles, responsibilities, and duties of the pharmacist. Pharmacy (Basel) 2020 Aug 07;8(3):140 [FREE Full text] [doi: 10.3390/pharmacy8030140] [Medline: 32784696]

23. Coughlan C, Nafde C, Khodatars S, Jeanes A, Habib S, Donaldson E, et al. COVID-19: lessons for junior doctors redeployed to critical care. Postgrad Med J 2021 Mar;97(1145):188-191 [FREE Full text] [doi: 10.1136/postgradmedj-2020-138100] [Medline: 32581082]

24. Levesque J, Harris MF, Russell G. Patient-centred access to health care: conceptualising access at the interface of health systems and populations. Int J Equity Health 2013 Mar 11;12(1):18 [FREE Full text] [doi: 10.1186/1475-9276-12-18] [Medline: 23496984]

25. Gandhi S. Will Health Care Infrastructure Survive the COVID-19 Pandemic? justanoldcountrydoctor. 2020 Apr 14. URL: https://justanoldcountrydoctor.com/2020/04/14/will-health-care-infrastructure-survive-the-covid-19-pandemic/ [accessed 2020-07-30] 
26. Kohli P, Virani SS. Surfing the Waves of the COVID-19 Pandemic as a Cardiovascular Clinician. Circulation 2020 Jul 14;142(2):98-100. [doi: 10.1161/CIRCULATIONAHA.120.047901] [Medline: $\underline{32369419}$ ]

27. Marshall E, Breton M, Green M, Miller L, Smithman M, Frymire E. [Poster] The CUP Study: Examining the effectiveness of centralized waitlists in Connecting Unattached Patients to primary care providers. Virtually CAHSPR. 2020. URL: https:/ /cahspr.ca/wp-content/uploads/2020/06/Book-of-Abstracts-Virtually-CAHSPR-2020.pdf [accessed 2021-09-28]

28. Buchan SA, Rosella LC, Finkelstein M, Juurlink D, Isenor J, Marra F, Public Health Agency of Canada/Canadian Institutes of Health Research Influenza Research Network (PCIRN) Program DeliveryEvaluation Group. Impact of pharmacist administration of influenza vaccines on uptake in Canada. CMAJ 2017 Jan 30;189(4):E146-E152 [FREE Full text] [doi: 10.1503/cmaj.151027] [Medline: 27503864]

29. Beahm NP, Smyth DJ, Tsuyuki RT. Outcomes of Urinary Tract Infection Management by Pharmacists (ROUTMAP): A study of pharmacist prescribing and care in patients with uncomplicated urinary tract infections in the community. Can Pharm J (Ott) 2018 Jun 03;151(5):305-314 [FREE Full text] [doi: 10.1177/1715163518781175] [Medline: 31080530]

30. Tsuyuki RT, Houle SK, Charrois TL, Kolber MR, Rosenthal MM, Lewanczuk R, RxACTION Investigators. Randomized Trial of the Effect of Pharmacist Prescribing on Improving Blood Pressure in the Community: The Alberta Clinical Trial in Optimizing Hypertension (RxACTION). Circulation 2015 Jul 14;132(2):93-100. [doi: 10.1161/CIRCULATIONAHA.115.015464] [Medline: 26063762]

31. Al Hamarneh YN, Charrois T, Lewanczuk R, Tsuyuki RT. Pharmacist intervention for glycaemic control in the community (the RxING study). BMJ Open 2013 Sep 24;3(9):e003154 [FREE Full text] [doi: 10.1136/bmjopen-2013-003154] [Medline: 24068762]

32. Health system governance. World Health Organization. URL: https://www.who.int/topics/health policy/en/ [accessed 2020-07-30]

33. Tomoaia-Cotisel A, Scammon DL, Waitzman NJ, Cronholm PF, Halladay JR, Driscoll DL, et al. Context matters: the experience of 14 research teams in systematically reporting contextual factors important for practice change. Ann Fam Med 2013 May 20;11 Suppl 1(Suppl_1):S115-S123 [FREE Full text] [doi: 10.1370/afm.1549] [Medline: 23690380]

34. Mason M. Sample size and saturation in $\mathrm{PhD}$ studies using qualitative interviews. Forum qualitative Sozialforschung/Forum: qualitative social research 2010;11(3). [doi: 10.17169/fqs-11.3.1428]

35. Sandelowski M. Sample size in qualitative research. Res Nurs Health 1995 Apr;18(2):179-183. [doi: 10.1002/nur.4770180211] [Medline: 7899572]

36. Morse J. Designing funded qualitative research. In: Denzin NK, Lincoln YS, editors. Handbook of Qualitative Inquiry. Thousand Oaks, CA: Sage Publications, Inc; 1994:220-235.

37. Gale NK, Heath G, Cameron E, Rashid S, Redwood S. Using the framework method for the analysis of qualitative data in multi-disciplinary health research. BMC Med Res Methodol 2013 Sep 18;13(1):117 [FREE Full text] [doi: 10.1186/1471-2288-13-117] [Medline: 24047204]

38. Srivastava A, Thomson S. Framework analysis: a qualitative methodology for applied policy research. Journal of Administration and Governance 2009 Jan 02;4(2) [FREE Full text]

39. Puhl RM, Peterson JL, Luedicke J. Parental perceptions of weight terminology that providers use with youth. Pediatrics 2011 Oct;128(4):e786-e793 [FREE Full text] [doi: 10.1542/peds.2010-3841] [Medline: 21949145]

40. Fetters MD, Curry LA, Creswell JW. Achieving integration in mixed methods designs-principles and practices. Health Serv Res 2013 Dec;48(6 Pt 2):2134-2156 [FREE Full text] [doi: 10.1111/1475-6773.12117] [Medline: 24279835]

41. Goldsmith L, Marshall E. Implementing a fully integrated mixed methods design with a cross-national, multidisciplinary team: the Early Career Primary Care (ECPC) Study. Implementing a fully integrated mixed methods design with a cross-national, multidisciplinary team; 2019 Presented at: Canadian Association for Health Services and Policy Research (CAHSPR) Annual Conference; May 29-31, 2019; Halifax, NS URL: https://cahspr.ca/wp-content/uploads/2020/11/ Book-of-Abstracts-CAHSPR-2019.pdf

42. Problems Coordinating and Accessing Primary Care for Attached and Unattached Patients in a Pandemic Year. A Longitudinal Mixed Methods Study with Rapid Reporting and Planning for the Road Ahead. PUPPY Study - Dr Emily Gard Marshall, PhD. URL: https://www.emilygardmarshall.ca/puppy.html [accessed 2021-08-31]

\section{Abbreviations}

CIHR: Canadian Institutes of Health Research

CUP: Comparative analysis of centralized waitlist effectiveness, policies, and innovations for connecting unattached patients to primary care providers

PIHCI: Primary and Integrated Healthcare Innovations

PUPPY: Problems Coordinating and Accessing Primary Care for Attached and Unattached Patients Exacerbated During the COVID-19 Pandemic Year

SPOR: Strategy for Patient Oriented Research 
Edited by G Eysenbach; submitted 27.04.21; peer-reviewed by I Idris, P Ahmad; comments to author 31.08.21; revised version received 09.09.21; accepted 09.09.21; published 13.10.21

Please cite as:

Marshall EG, Breton M, Cossette B, Isenor J, Mathews M, Ayn C, Smithman MA, Stock D, Frymire E, Edwards L, Green M

Problems in Coordinating and Accessing Primary Care for Attached and Unattached Patients Exacerbated During the COVID-19

Pandemic Year (the PUPPY Study): Protocol for a Longitudinal Mixed Methods Study

JMIR Res Protoc 2021;10(10):e29984

URL: https://www.researchprotocols.org/2021/10/e29984

doi: $\underline{10.2196 / 29984}$

PMID: $\underline{34559672}$

CEmily Gard Marshall, Mylaine Breton, Benoit Cossette, Jennifer Isenor, Maria Mathews, Caitlyn Ayn, Mélanie Ann Smithman, David Stock, Eliot Frymire, Lynn Edwards, Michael Green. Originally published in JMIR Research Protocols (https://www.researchprotocols.org), 13.10.2021. This is an open-access article distributed under the terms of the Creative Commons Attribution License (https://creativecommons.org/licenses/by/4.0/), which permits unrestricted use, distribution, and reproduction in any medium, provided the original work, first published in JMIR Research Protocols, is properly cited. The complete bibliographic information, a link to the original publication on https:/www.researchprotocols.org, as well as this copyright and license information must be included. 